\title{
Recombinant Factor VIla Use for Endoscopic Retrograde Cholangiopancreatography With Sphincterotomy in a Patient With Choledocholithiasis and Unusual Coagulopathy
}

\author{
Molham Abdulsamad $^{\mathrm{a}, \mathrm{b}}$, Pavithra Reddy ${ }^{\mathrm{a}}$, Suvarna Guvvala ${ }^{\mathrm{a}}$, Anil Dev ${ }^{\mathrm{a}}$
}

\begin{abstract}
Endoscopic retrograde cholangiopancreatography (ERCP) is a procedure that combines the use of endoscopy and fluoroscopy to diagnose and treat pancreaticobiliary disorders. The risks of ERCP include pancreatitis, infection, bleeding and perforation. Bleeding during ERCP typically develops after sphincterotomy, hence patients should be screened and tested for coagulopathy before undergoing ERCP. Coagulopathy is a major risk factor for ERCP-related bleeding. Inherited factor VII (FVII) deficiency is a rare autosomal recessive hemorrhagic disorder that can lead to significant coagulopathy and severe bleeding if not appropriately recognized and treated preoperatively. Clinically, the disease ranges between an asymptomatic state to lethal hemorrhage and the degree of FVII deficiency does not correlate with the severity of bleeding. The use of FVII replacement therapy has been reported to prevent bleeding during surgery. We present the first report of a patient with a rare cause of coagulopathy due to inherited FVII deficiency who successfully underwent ERCP with sphincterotomy without bleeding where we used recombinant factor VIIa before and after the procedure.
\end{abstract}

Keywords: Recombinant factor VIIa; NovoSeven; ERCP with sphincterotomy; Choledocholithiasis; Coagulopathy

\section{Introduction}

Endoscopic retrograde cholangiopancreatography (ERCP) is a procedure that combines the use of endoscopy and fluoroscopy to diagnose and treat pancreaticobiliary disorders. Jaundice as a result of biliary obstruction due to choledocholithiasis is one of the most common indications to perform ERCP with an intent to perform endoscopic sphincterotomy to facilitate stone removal. The risks of ERCP include pancreatitis, infection, bleeding and

Manuscript accepted for publication March 01, 2017

aDepartment of Medicine, Division of Gastroenterology, Bronx Lebanon Hospital Center, 1650 Selwyn Avenue, Suite\# 10C, Bronx, NY 10457, USA

${ }^{b}$ Corresponding Author: Molham Abdulsamad, Department of Medicine, Bronx Lebanon Hospital Center, 1650 Selwyn Ave, Suite \#10C, Bronx, NY 10457, USA.Email: MABDULSA@bronxleb.org

doi: https://doi.org/10.14740/gr812w perforation [1]. Bleeding during ERCP typically develops after sphincterotomy, hence patients should be screened and tested for coagulopathy before undergoing ERCP. Clinically evident bleeding occurs in $0.1-2 \%$ of sphincterotomies [2]. The presence of any coagulopathy, thrombocytopenia or the use of anticoagulantion therapy are the most important risk factors for bleeding after sphincterotomy. Inherited factor VII (FVII) deficiency is a rare autosomal recessive hemorrhagic disorder that can lead to significant coagulopathy and severe bleeding if not appropriately recognized and treated preoperatively. The estimated prevalence of inherited FVII deficiency is 1 in 500,000 [3].

The use of FVII replacement therapy has been reported to prevent bleeding during surgery in such patients and some reports had limited experience but successful use of recombinant activated FVII (rFVIIa) during a variety of surgical procedures including cesarean delivery, hysterectomy, coronary artery bypass grafting, thoracic surgery, and intracerebral hematoma surgery. There are no data published to date regarding experience with ERCP and sphincterotomy. Herein we present the first report of a patient with a rare cause of coagulopathy due to inherited FVII deficiency who successfully underwent ERCP with sphincterotomy without bleeding where we used recombinant factor VIIa before and after the procedure.

\section{Case Report}

A 21-year-old female with no known medical illness presented to our hospital with acute right upper quadrant abdominal pain for 2 days. She had no fever, chills or jaundice. She denied any history of easy bruising, recurrent epistaxis, excessive bleeding or menorrhagia. She denied smoking or alcohol use. Her physical exam revealed no scleral icterus and her abdominal exam revealed right upper quadrant tenderness, non-distended abdomen with no hepatosplenomegaly. Her laboratories were significant for leukocytosis with a white blood cell count of $14.8 \times 10^{3} / \mu \mathrm{L}$, hemoglobin of $12 \mathrm{~g} / \mathrm{dL}$, platelet count of 290 $\times 10^{3} / \mu \mathrm{L}$, and normal liver chemistry except for alkaline phosphatase of $102 \mathrm{IU} / \mathrm{L}$. Bleeding profile revealed a normal partial thromboplastin time and elevated prothrombin time (PT) of $25.3 \mathrm{~s}$ (normal range: $9.5-12 \mathrm{~s}$ ) and an international normalized ratio (INR) of 2.2 (normal range: 0 - 2.0). Ultrasound of the abdomen revealed cholelithiasis with pericholecystic fluid and gallbladder wall thickening concerning for acute cholecys- 


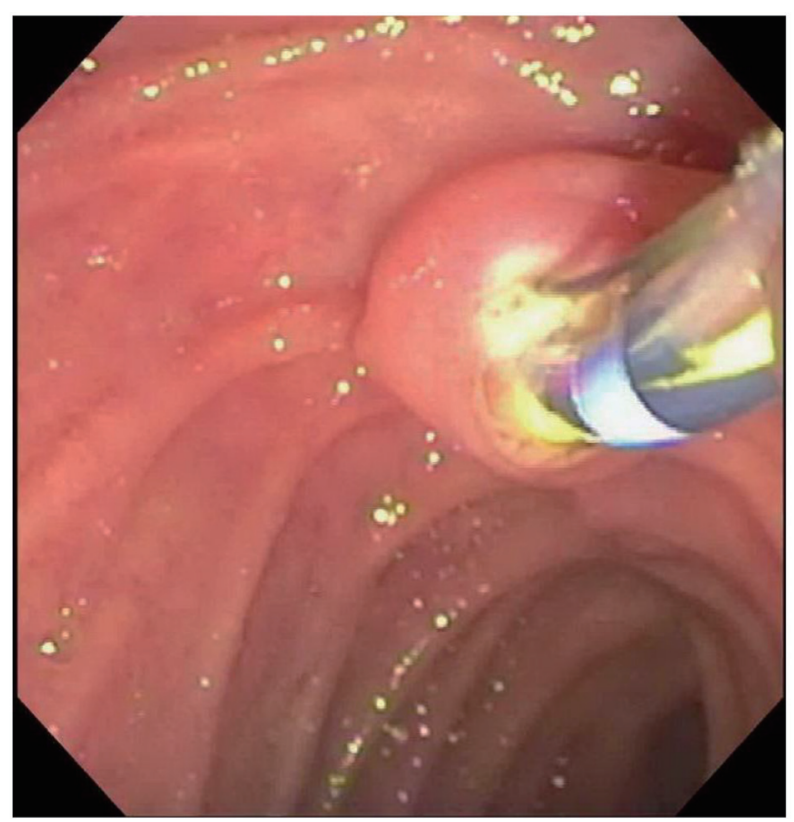

Figure 1. Endoscopic image of the ampulla while performing sphincterotomy with no bleeding.

titis. Common bile duct (CBD) diameter was $7 \mathrm{~mm}$. Magnetic resonance cholangiopancreatography (MRCP) revealed a calculus within the dilated CBD which was also confirmed by endoscopic ultrasound (EUS) examination. She was given two doses of injectable vitamin K $10 \mathrm{mg}$ and 5 units of fresh frozen plasma (FFP) over $48 \mathrm{~h}$ with no change in her elevated INR, hence a comprehensive workup for her coagulopathy was performed. This revealed a low factor VII to $34 \%$ (normal 60$150 \%$ ). Mixing study revealed correction of her PT indicating FVII deficiency rather than the presence of an inhibitor. There was no evidence of any underlying liver disease and no use of any anticoagulation therapy. We performed an ERCP after giving 2,000 $\mu \mathrm{g}(30 \mu \mathrm{g} / \mathrm{kg})$ of rFVIIa (NovoSeven $\left.{ }^{\circledR} \mathrm{RT}\right)$ immediately before the procedure, and a sphincterotomy was made with a Cook Tri-Tome sphincterotome (Cook America, Winston-Salem, NC) using ERBE ${ }^{\circledR}$ electrocautery (Fig. 1). There was no post-sphincterotomy bleeding and a stone was successfully removed from the bile duct. She received another dose of $2,000 \mu \mathrm{g}$ of rFVIIa $4 \mathrm{~h}$ after the procedure to ensure hemostasis and decrease the risk of post-procedural delayed bleeding. An INR of 1.2 was achieved after giving the second dose (Fig. 2). Her hemoglobin remained stable and did not require any blood transfusion. A few days later, she had successful laparoscopic cholecystectomy after giving rFVIIa before the procedure and no evidence of bleeding was noted during or after the surgery.

\section{Discussion}

A variety of inherited coagulation disorders are associated with clinical bleeding and FVII deficiency is considered a rare disease. FVII is typically synthesized in the liver and its part of the vitamin K-dependent coagulation factors. Activation of this factor will initiate coagulation via the extrinsic pathway which rapidly activates clotting factor $\mathrm{X}$ and initiates the coagulation cascade. It has a short half-life of $2-4 \mathrm{~h}$ and is primarily activated by tissue factor (factor III) in response to tissue injury. Genetically, more than 100 mutations have been identified in the FVII gene located on chromosome 13 [4]. Clinically, the disease ranges between an asymptomatic state to lethal hemorrhage and the degree of FVII deficiency does not correlate with the severity of bleeding. Acquired FVII deficiency can be seen in patients receiving warfarin, patients with liver cirrhosis, vitamin K deficiency and rarely due to acquired inhibitors to FVII.

The total number of FVII-deficient patients worldwide is low which explains the lack of large-scale studies in this field. The most predominant feature of the disease is bleeding with variable onset, site, and severity. The patient may present with epistaxis, hemarthrosis, menorrhagia or excessive bleeding with trauma or invasive procedures. Other features include pregnancy loss, impaired wound healing and interestingly thrombosis with a multifactorial etiology but more attributable to therapy rather than the underlying factor deficiency. Typically the patient will have prolonged PT and elevated INR with normal aPTT. Factor activity level below $50 \%$ of normal is generally considered as a deficiency. Other diagnostic evaluations include mixing study, thrombin generation test and FVII antigen. None of the above

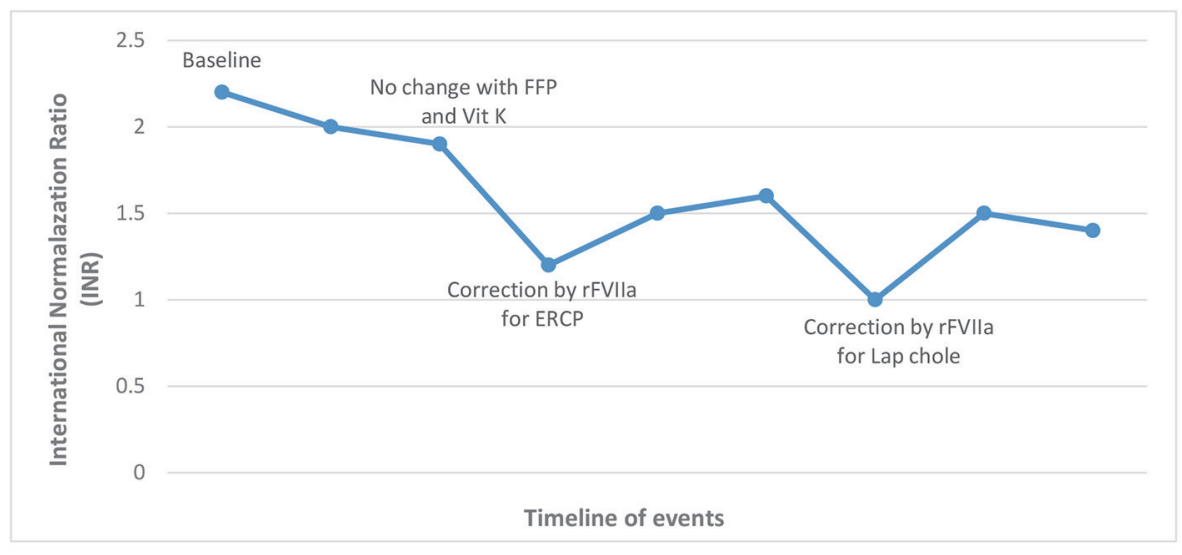

Figure 2. INR level relative to rFVIla use. 
investigations can predict the perioperative risk of bleeding in these patients [5]. The level of FVII does not always correlate with the risk of bleeding. The risk of bleeding usually increases with levels of less than $10 \%$ and those with $>20 \%$ remain frequently asymptomatic. However, patients with levels $>20 \%$ may bleed and those with $<1 \%$ may remain asymptomatic [6].

There are various treatment options to treat hemorrhagic complications which can be also used for prophylaxis prior to surgical intervention and includes administration of fresh frozen plasma (FFP), prothrombin complex concentrate (PCC), FVII concentrate and rFVIIa. The best available option is rFVIIa but it is expensive compared to other options and has limited availability and risk of thrombosis remains a concern. rFVIIa has been used since 1988 for treating patients with severe hemophilia A [7]. Recently, its use for patients with FVII deficiency is rising. Approximately 30 - 40 case reports of perioperative rFVIIa use were reported [8]. It can be administered prior to surgery either as a bolus dose or continuous infusion [9]. There are few trials comparing the efficacy, adverse events and cost effectiveness of PCC and rFVIIa where the latter was superior. Adverse events associated with rFVIIa administration include the development of inhibitors against FVII and thrombotic events [10]. Recommended dosing of rFVIIa is 15 - $30 \mu \mathrm{g} / \mathrm{kg}$ IV bolus, repeated q4-6hr until hemostasis achieved during surgery. FFP is the least effective therapy and carries risks of transfusion reactions and infections, volume overload and there may be a substantial delay in achieving hemostatic levels of FVII. In this patient, 5 units of FFP were given over $48 \mathrm{~h}$ but without improving her INR preoperatively.

In this case, our patient had FVII deficiency with FVII level of $34 \%$ and an INR of 2.2. She underwent ERCP with successful sphincterotomy after receiving rFVIIa immediately before the procedure and at $4 \mathrm{~h}$ after the procedure to ensure hemostasis and decrease the risk of post-procedural delayed bleeding. She had no evidence of bleeding or thrombosis after the use of rFVIIa.

\section{Author Contributions}

All authors have made contributions to the article and have reviewed it before submission and agree to its publication in this form.

\section{Conflicts of Interest}

All authors declare that there is no conflict of interest regard- ing the publication of this paper.

\section{Consent}

Informed consent was obtained for this case report.

\section{References}

1. Cotton PB, Garrow DA, Gallagher J, Romagnuolo J. Risk factors for complications after ERCP: a multivariate analysis of 11,497 procedures over 12 years. Gastrointest Endosc. 2009;70(1):80-88.

2. Freeman ML, Nelson DB, Sherman S, Haber GB, Herman ME, Dorsher PJ, Moore JP, et al. Complications of endoscopic biliary sphincterotomy. $\mathrm{N}$ Engl J Med. 1996;335(13):909-918.

3. Mannucci PM, Duga S, Peyvandi F. Recessively inherited coagulation disorders. Blood. 2004;104(5):1243-1252.

4. Mariani G, Herrmann FH, Dolce A, Batorova A, Etro D, Peyvandi F, Wulff K, et al. Clinical phenotypes and factor VII genotype in congenital factor VII deficiency. Thromb Haemost. 2005;93(3):481-487.

5. Giansily-Blaizot M, Verdier R, Biron-Adreani C, Schved JF, Bertrand MA, Borg JY, Le Cam-Duchez V, et al. Analysis of biological phenotypes from 42 patients with inherited factor VII deficiency: can biological tests predict the bleeding risk? Haematologica. 2004;89(6):704-709.

6. de Moerloose P, Schved JF, Nugent D. Rare coagulation disorders: fibrinogen, factor VII and factor XIII. Haemophilia. 2016;22(Suppl 5):61-65.

7. Hedner U, Glazer S, Pingel K, Alberts KA, Blomback M, Schulman S, Johnsson H. Successful use of recombinant factor VIIa in patient with severe haemophilia A during synovectomy. Lancet. 1988;2(8621):1193.

8. Brenner B, Wiis J. Experience with recombinant-activated factor VII in 30 patients with congenital factor VII deficiency. Hematology. 2007;12(1):55-62.

9. Schulman S, Bech Jensen M, Varon D, Keller N, Gitel $\mathrm{S}$, Horoszowski H, Heim M, et al. Feasibility of using recombinant factor VIIa in continuous infusion. Thromb Haemost. 1996;75(3):432-436.

10. Neufeld EJ, Negrier C, Arkhammar P, Benchikh el Fegoun $\mathrm{S}$, Simonsen MD, Rosholm A, Seremetis S. Safety update on the use of recombinant activated factor VII in approved indications. Blood Rev. 2015;29(Suppl 1):S34-41. 